\title{
Prognostic impact of carbohydrate sulfotransferase 15 in patients with pancreatic ductal adenocarcinoma
}

\author{
ZENSHO ITO $^{1}$, KAZUKI TAKAKURA ${ }^{1}$, MACHI SUKA ${ }^{2}$, TOMOYA KANAI ${ }^{1}$, \\ RYOTA SAITO $^{3}$, SHUICHI FUJIOKA ${ }^{3}$, MIKIO KAJIHARA ${ }^{1}$, HIROYUKI YANAGISAWA ${ }^{2}$, \\ TAKEYUKI MISAWA ${ }^{3}$, TADASHI AKIBA ${ }^{3}$, SHIGEO KOIDO ${ }^{1,4}$ and TOSHIFUMI OHKUSA ${ }^{1,4}$ \\ ${ }^{1}$ Division of Gastroenterology and Hepatology, Department of Internal Medicine, \\ Jikei University School of Medicine, Kashiwa Hospital, Kashiwa, Chiba 277-8564; \\ ${ }^{2}$ Department of Public Health and Environmental Medicine, Jikei University School of Medicine, \\ Tokyo 105-8461; ${ }^{3}$ Department of Surgery, Jikei University School of Medicine, Kashiwa Hospital; \\ ${ }^{4}$ Institute of Clinical Medicine and Research, Jikei University School of Medicine, Kashiwa, Chiba 277-8564, Japan
}

Received September 8, 2016; Accepted February 27, 2017

DOI: $10.3892 / 01.2017 .6071$

\begin{abstract}
Patients with pancreatic ductal adenocarcinoma (PDA) typically succumb to mortality early, even following surgical resection. Therefore, prognostic factors associated with early mortality are required to improve the survival of patients with PDA following surgical resection. Carbohydrate sulfotransferase 15 (CHST15) is responsible for the biosynthesis of sulfated chondroitin sulfate E (CS-E), which serves a pivotal function in cancer progression by cleaving CD44. CHST15 and CD44 expression in PDA tissue were assessed as a prognostic factor in patients with PDA following surgical resection. A total of 36 consecutive patients with PDA were enrolled following surgical resection between January 2008 and December 2014. The intensities of CHST15 and CD44 expression were analyzed by immunohistochemical staining. The recurrence period was significantly earlier in the strong CHST15 expression group compared with the negative-to-moderate CHST15 expression group. Overall survival (OS) was also significantly decreased in the strong CHST15 expression group compared with the negative-to-moderate CHST15 expression group. Multivariate analysis also indicated significant associations between CHST15 overexpression and disease-free survival (DFS) and OS. However, expression of CD44 in PDA tissue was not associated with DFS or OS. The present study has demonstrated for the first time that high CHST15 expression in PDA tissue
\end{abstract}

Correspondence to: Dr Shigeo Koido or Dr Kazuki Takakura, Division of Gastroenterology and Hepatology, Department of Internal Medicine, Jikei University School of Medicine, Kashiwa Hospital, 163-1 Kashiwa-shita, Kashiwa, Chiba 277-8564, Japan

E-mail: shigeo_koido@jikei.ac.jp

E-mail: ktakakura@jikei.ac.jp

Key words: pancreatic ductal adenocarcinoma, CHST15, CD44, recurrence, prognostic marker, surgical resection may represent a potential predictive marker of DFS and OS in patients with PDA following surgical resection.

\section{Introduction}

The most common type of pancreatic tumor is pancreatic ductal adenocarcinoma (PDA), which arises from the exocrine component of the pancreas $(1,2)$. Although surgical resection is the only curative treatment for PDA, $<20 \%$ of patients with PDA are able to undergo surgery (3). Despite improved surgical techniques, chemotherapeutic agents and/or radiation therapy, patients with PDA have an overall 5-year survival rate of only $5 \%$ (4). Notably, a significant population of patients with PDA demonstrate early local recurrence and/or metastases following surgical resection (5). Therefore, improvement of early mortality following surgical resection for patients with PDA is urgently needed. One major focus of researchers has been to identify prognostic factors associated with early recurrence in patients with PDA following surgical resection.

Several previous studies have demonstrated the potential functions of chondroitin sulfate (CS), which is an important component of the extracellular matrix (ECM) in normal and tumor tissues (6-9). CS is a unique, highly sulfated sugar, and is abundantly expressed in the ECM of tumor cells, resulting in tumor development and progression $(10,11)$. CS has been classified into five different types due to the presence of differently sulfated disaccharide units: CS-A (4-sulfated), CS-B, (dermatan sulfate), CS-C (6-sulfated), CS-D (2,6-disulfated), and CS-E (4,6-disulfated) (10). The sulfation pattern is involved in cancer progression (12). In particular, CS-E, a highly sulfated glycosaminoglycan, is expressed in the ECM of adenocarcinoma (12) and is involved in tumor proliferation, metastasis and angiogenesis $(13,14)$. The biosynthesis of CS-E is mediated by $\mathrm{N}$-acetylgalactosamine 4 -sulfate 6-O-sulfotransferase [GalNAc4S-6ST, also known as carbohydrate sulfotransferase 15 (CHST15)], which transfers sulfate from 3'-phosphoadenosine-5'-phosphosulfate to the 6-O of GalNAc4S on CS-A (15). A previous report indicated 
a direct association between CHST15 and the proliferation of human PDA cell lines in vivo and in vitro (16). Therefore, CHST15 levels in human PDA tissue may represent a potential prognostic biomarker. Furthermore, the products of CS-E degradation in certain tumor-associated ECM promote cell adhesion and migration by cleaving CD44 in PDA tissue (17). CD44 is a cell surface receptor for several ECM components and is also associated with tumor cell migration and metastasis $(18,19)$. Notably, the ectodomain of CD44 on tumor cells is cleaved by multiple stimulations (20). The level of CD44 cleavage and circulating soluble CD44 is involved in tumor migration and invasion (21). In addition, the cleaved intracellular domain of CD44 activates stemness factors, including Nanog homeobox, sex determining region Y-box 2, and POU class 5 homeobox 1, and contributes to tumorigenesis (22). Overexpression of CD44 in the presence of CS-E may be associated with enhanced amounts of CD44 cleavage and result in early recurrence in patients with PDA following surgical resection $(17,20)$. These reports indicated the potential of CHST15 and CD44 as prognostic factors in patients with PDA following surgical resection. Previous reports have indicated that low neutrophil-to-lymphocyte ratios (NLRs) and low carbohydrate antigen 19-9 (CA19-9) levels may be associated with significantly improved prognoses in patients with PDA $(23,24)$. Thus, the present study analyzed NLRs and CA19-9 levels in the peripheral blood and the expression of CHST15 and CD44 in PDA tissue from surgical specimens as prognostic factors for patients with PDA following surgical resection. The major aim of the present study was to identify prognostic factors associated with early recurrence in patients with PDA following surgical resection. Overexpression of CHST15 in PDA tissue was demonstrated to be significantly associated with shorter disease-free survival (DFS) and overall survival (OS) in patients with PDA following surgical resection.

\section{Materials and methods}

PDA samples from patients. The present study included patients with PDA who underwent macroscopically curative resection by total pancreatectomy, pancreaticoduodenectomy or pylorus-preserving pancreaticoduodenectomy with lymph node dissection at Jikei University Kashiwa Hospital (Kashiwa, Japan) between January 2008 and December 2014. Tumor samples from 36 consecutive patients with PDA were collected. Information regarding clinical features of the patients, including age, sex, tumor location, tumor differentiation and tumor recurrence, was obtained from medical records. All laboratory data were obtained around the time of surgery. OS was defined as the time from diagnosis to mortality from any cause. DFS was defined as the time from the date of surgery to the first radiological evidence of recurrence or mortality without evidence of recurrence or a second primary cancer. Pathological data, including tumor stage, grade, and size were obtained from surgical reports and assessed using the pancreatic cancer TNM staging system set forth by the American Joint Committee on Cancer (AJCC) (25). The baseline characteristics of patients with PDA are presented in Table I. The present study was reviewed and approved by the Ethics Committee of Jikei University School of Medicine
(Tokyo, Japan) and by the clinical study committee of Jikei University Kashiwa Hospital (grant no. 26-370, 7876). The review board approved the present investigation and waived the need for written informed consent from study participants due to the retrospective, non-interventional nature of this study. Study procedures were conducted in accordance with the Helsinki Declaration.

Laboratory data. All laboratory data, including levels of CA19-9, neutrophils, lymphocytes, leukocytes, platelets (PLTs), hemoglobin ( $\mathrm{Hb}$ ), albumin (Alb), and C-reactive protein (CRP), were obtained around the time of surgery. NLR was calculated by dividing the neutrophil count by the lymphocyte count. All data were assessed to determine the prognostic impact of the examined metrics on patients with PDA following surgical resection.

Immunohistochemical staining. Staining for CHST15 and human CD44 was performed on primary tumor samples from patients with PDA. Formalin-fixed, paraffin-embedded sections (thickness, $6 \mu \mathrm{m}$ ) were stained with hematoxylin and eosin for histopathological evaluation. All immunohistochemical staining of tumor sections (size, 20x30 mm) was performed on formalin-fixed and paraffin-embedded sections. Briefly, the sections were incubated with goat anti-human CHST15 antibody (1:200; cat. no. AF3365; R\&D Systems, Inc., Minneapolis, MN, USA) for $1 \mathrm{~h}$ at room temperature or rabbit anti-human CD44 antibody (1:500; cat. no. ab41478, Abcam, Cambridge, MA, USA) for $1 \mathrm{~h}$ at room temperature, and washed with phosphate-buffered solution (PBS). For CHST15 staining, the sections were incubated for $30 \mathrm{~min}$ at room temperature with rabbit anti-goat IgG-biotin $(67 \mu \mathrm{g} / \mathrm{ml}$; cat. no. ab6741, Abcam). For CD44 staining, the sections were incubated for $30 \mathrm{~min}$ at room temperature with goat anti-rabbit IgG-biotin (26.7 $\mu \mathrm{g} / \mathrm{ml}$; cat. no. 111-036-045; Jackson ImmunoResearch Laboratories, Inc., West Grove, PA, USA). The IgG labeled-sections were incubated for $30 \mathrm{~min}$ at room temperature, with horseradish peroxidase-conjugated streptavidin, and developed with 3,3'-diaminobenzidine (Nichirei Biosciences Inc., Tokyo, Japan). The nuclei were subsequently counterstained with hematoxylin. The assessment of CHST15 or CD44 expression was performed in five random fields within the tumor under a light microscope (magnification, x100). CHST15 and CD44 staining statuses were graded as follows: 1, Negative, no staining or little staining in $\leqq 20 \%$ of PDA tissue; 2 , Weak, weak staining in $>80 \%$ of PDA tissue; 3 , Moderate, moderate staining in $>80 \%$ of PDA tissue; and 4 , Strong, strong or complete staining in $>80 \%$ of PDA tissue (26). CHST15 and CD44 expression levels were independently interpreted by three investigators who were blinded to the clinical information of the patients. The negative control was stained using an identical procedure but with the primary antibody omitted.

Statistical analysis. All statistical analyses were performed using SAS software, version 9.4 (SAS Institute, Inc., Cary, NC, USA). Categorical variables were compared using the $\chi^{2}$ test. Continuous variables were compared using the Mann-Whitney $\mathrm{U}$ test. DFS and OS were estimated using the Kaplan-Meier method, and differences were measured using log-rank tests. 
Table I. Baseline characteristics of patients with pancreatic cancer.

\begin{tabular}{lrr}
\hline Clinical characteristic & $\mathrm{n}$ & $(\%)$ \\
\hline Age at surgery & & \\
$\quad<65$ years & 12 & 33.3 \\
$\geq 65$ years & 24 & 66.7 \\
Sex & & \\
Male & 20 & 55.6 \\
Female & 16 & 44.4 \\
Tumor location & & \\
Head & 30 & 83.3 \\
Body-to-tail & 6 & 16.7 \\
Pathology & & 77.8 \\
Well-to-moderate & 28 & 22.2 \\
Poor & 8 & \\
Tumor stage & & 91.7 \\
I/II & 33 & 27.8 \\
III/IV & 3 & 72.2 \\
Tumor size & & \\
$<3$ cm & 10 & \\
$\geq 3$ cm & 26 & \\
\hline
\end{tabular}

A multivariate Cox hazard regression model was used to examine independent predictors of DFS and OS. $\mathrm{P}<0.05$ was considered to indicate a statistically significant difference.

\section{Results}

PDA patient characteristics. A total of 36 consecutive patients with PDA were enrolled in the present study, and their clinical characteristics are summarized in Table I. The numbers of male and female patients were 20 (55.6\%) and 16 (44.4\%), respectively. The median patient age at diagnosis was 65.6 years (range, 44-79 years). There were 3, 30, and 3 patients with PDA classified into AJCC stages I, II, and III, respectively. The median DFS of the patients was 310 days (range, 62-2050 days), and the median OS was 512.5 days (range, 216-2296 days). The majority of tumors $(n=30,83.3 \%)$ were located in the head of the pancreas. In addition, all tumors were confirmed to be associated with invasive ductal adenocarcinoma.

Laboratory data. All laboratory data were obtained by exploration around the time of surgery and assessed for prognostic impact on patients with PDA following surgical resection. As expected, pathology, tumor stage, and low NLRs in patients with PDA were significantly associated with improved DFS (Table II). In addition, pathology, low NLRs and high Alb levels in patients with PDA were significantly associated with longer OS in the present study. In contrast, laboratory data, including leukocyte and PLT counts and Hb and CRP levels, were not associated with DFS or OS (Tables II and III).

CHST15 expression in PDA tissue. To assess the significance of CHST15 expression on PDA tissue as a prognostic factor in patients with PDA following surgical resection, surgical specimens were examined by immunohistochemical staining (Fig. 1). CHST15 expression was detected in the cytoplasm and membrane of PDA cells. All patients with PDA were divided into two groups according to CHST15 expression in the PDA tissue as follows: A strong CHST15 expression group and a negative-to-moderate CHST15 expression group. The 36 patients with PDA included 8 patients $(22.2 \%)$ with strong CHST15 expression and 28 patients $(77.8 \%)$ with negative-to-moderate CHST15 expression. Of the 36 patients, 4 patients with PDA (11.1\%) demonstrated negative CHST15 expression in the present study. There were no significant differences in sex, age at surgery, tumor location, tumor stage, tumor size or tumor pathology between the strong and negative-to-moderate CHST15 expression groups (Table IV). Kaplan-Meier curves for PDA DFS and OS in patients following surgery are presented in Fig. 2A. Notably, the recurrence period was significantly shorter in the strong CHST15 expression group compared with the negative-to-moderate expression group $(\mathrm{P}=0.014)$. Furthermore, OS was significantly shorter in the strong CHST15 expression group compared with the negative-to-moderate expression group $(\mathrm{P}=0.022)$. The multivariate analysis also indicated a significant association of DFS with CHST15 expression $[\mathrm{P}<0.001$; hazard ratio (HR), 9.456; 95\% confidence interval (CI), 2.644-33.815] and of OS with CHST15 expression ( $\mathrm{P}=0.012$; HR, 3.690; $95 \%$ CI, 1.331-10.231). The median DFS was 160.5 days in the strong CHST15 expression group and 351 days in the negative-to-moderate CHST15 expression group (Fig. 2A). In contrast, the median OS was 426 days in the strong CHST15 expression group and 594.5 days in the negative-to-moderate CHST15 expression group (Fig. 2B). In addition, no CHST15 staining positivity was detected in cells when omitting the first antibody, supporting the specificity of the staining. These results indicated that strong CHST15 expression may be a potential prognostic factor for patients with PDA following surgery.

CD44 expression on PDA tissue. CS-E degradation products that are mediated by CHST15 (15) have previously been reported to promote tumor cell adhesion and migration by cleaving CD44 in PDA tissue (17). The CD44 antibody used in the present study was generated from an immunogen sequence, DHTKQNQDWTQWNPSHSN, located within exon 8 of CD44 isoform 1. This epitope is present in the CD44 epican of tumor cells, but not in CD44H or CD44E $(27,28)$. CD44 expression was detected in the cytoplasm and membrane of PDA cells (Fig. 3). CD44 expression status was also assessed as negative, weak, moderate, or strong. Of the 36 patients, $17(47.2 \%), 5(13.9 \%)$, and $9(25.0 \%)$ had surgical specimens exhibiting weak, moderate, and strong CD44 expression, respectively (Fig. 3). Of the 36 patients, 5 patients with PDA $(13.9 \%)$ were negative for CD44 expression in the present study. No association was observed between CD44 expression levels in PDA tissue and progression, defined as DFS and OS $(\mathrm{P}=0.598$ and 0.326 , respectively). Furthermore, to evaluate the relationship between CHST15 and CD44, the same immunohistochemical stained areas were compared in PDA tissue. PDA cells expressing high levels of CHST15 did not necessarily exhibit high CD44 expression levels. In addition, no CD44 staining positivity in cells was detected when omitting the first antibody, supporting the specificity of the staining. 
Table II. Multivariate analysis of disease-free survival.

\begin{tabular}{lllr}
\hline Characteristic & HR & 95\% CI & P-value \\
\hline Age at surgery ( $\geq 65$ years vs. $<65$ years) & 0.615 & $0.213-1.773$ & 0.368 \\
Tumor location (body-to-tail vs. head) & 0.237 & $0.056-1.001$ & 0.050 \\
Pathology (poor vs. well-to-moderate) & 2.952 & $1.033-8.435$ & 0.043 \\
Tumor stage (III/IV vs. I/II) & 0.179 & $0.035-0.909$ & 0.038 \\
Leukocytes & 1.000 & $1.000-1.000$ & 0.891 \\
NLR & 1.385 & $1.098-1.748$ & 0.006 \\
CRP levels & 0.783 & $0.445-1.380$ & 0.398 \\
Hemoglobin levels & 1.096 & $0.700-1.714$ & 0.689 \\
Platelet levels & 1.021 & $0.972-1.072$ & 0.412 \\
Albumin levels & 0.657 & $0.267-1.619$ & 0.362 \\
CA19-9 levels & 1.000 & $1.000-1.000$ & 0.914 \\
CHST15 (strong vs. negative-to-moderate) & 9.456 & $2.644-33.815$ & $<0.001$ \\
\hline
\end{tabular}

HR, hazard ratio; CI, confidence interval; NLR, neutrophil-to-lymphocyte ratio; CRP, C-reactive protein; CA19-9, carbohydrate antigen 19-9; CHST15, carbohydrate sulfotransferase 15 .

Table III. Multivariate analysis of overall survival.

\begin{tabular}{llll}
\hline Characteristic & HR & 95\% CI & P-value \\
\hline Age at surgery ( $\geq 65$ years vs. $<65$ years) & 0.380 & $0.125-1.160$ & 0.089 \\
Tumor location (body-to-tail vs. head) & 1.783 & $0.472-6.746$ & 0.394 \\
Pathology (poor vs. well-to-moderate) & 3.169 & $1.074-9.349$ & 0.037 \\
Tumor stage (III/IV vs. I/II) & 0.588 & $0.187-1.848$ & 0.364 \\
Leukocytes & 1.000 & $1.000-1.000$ & 0.790 \\
NLR & 1.279 & $1.014-1.615$ & 0.038 \\
CRP levels & 0.690 & $0.347-1.374$ & 0.291 \\
Hemoglobin levels & 0.750 & $0.482-1.165$ \\
Platelet levels & 1.040 & $0.987-1.096$ \\
Albumin levels & 0.420 & $0.178-0.995$ & 0.201 \\
CA19-9 levels & 1.000 & $1.000-1.001$ & 0.140 \\
CHST15 (strong vs. negative-to-moderate) & 3.690 & $1.331-10.231$
\end{tabular}

HR, hazard ratio; CI, confidence interval; NLR, neutrophil-to-lymphocyte ratio; CRP, C-reactive protein; CA19-9, carbohydrate antigen 19-9; CHST15, carbohydrate sulfotransferase 15 .

\section{Discussion}

In the present study, CHST15 and CD44 intensity was analyzed in PDA surgical specimens and associations with the prognosis, defined as DFS and OS, were assessed in patients following surgical resection. Furthermore, the laboratory data concerning the peripheral blood, obtained around the time of surgery, were assessed as prognostic markers. Significantly prolonged DFS and OS were observed in patients with PDA without strong expression of CHST15. Furthermore, low NLRs were significantly associated with prolonged DFS and OS in the present study. Thus, the data presented herein suggested that high CHST15 expression in PDA tissue and high NLRs may be recurrence markers in patients with PDA following surgery.
It has previously been demonstrated that tumor cell-derived CS-E is mediated by CHST15 and is involved in the various stages of tumor progression and invasion (14). Human PDA tissue has been successfully treated in nude mice using small interfering RNA-based selective silencing of the CHST15 gene to selectively inhibit CS-E expression (16). Therefore, CHST15/CS-E axis-mediated PDA cell proliferation may be a therapeutic target for the early progression of PDA in humans. As CHST15 expression is significantly associated with prognosis in patients with PDA following surgical resection, CHST15 may be a novel therapeutic target. These reports led to the assessment of CHST15 expression in surgical specimens as a prognostic marker in patients with PDA following surgery in the present study. A total of 36 consecutive patients with PDA were classified into two types based on CHST15 
(A)

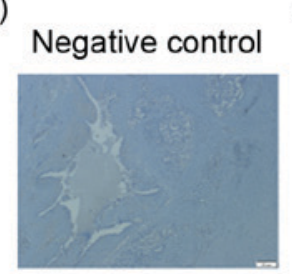

(F)

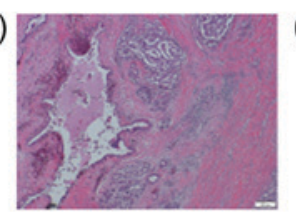

(B)

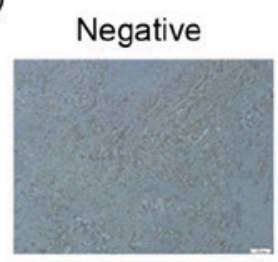

(G)

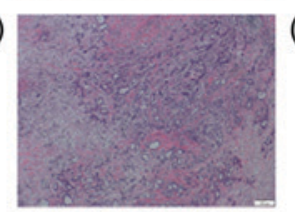

(C)

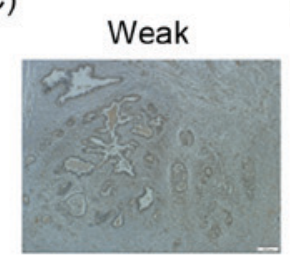

(H)

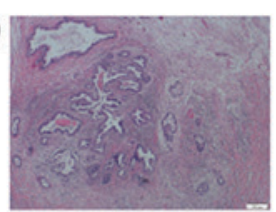

(D)
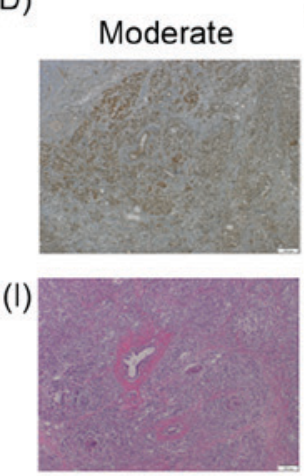

(E)

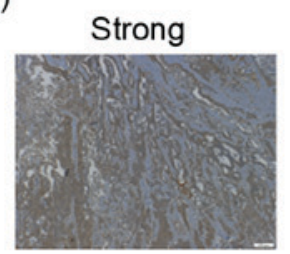

(J)

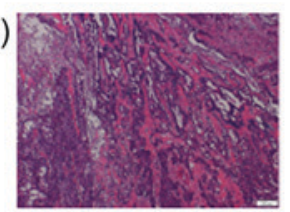

Figure 1. CHST15 intensity in PDA tissue. Representative images of PDA tissue stained with CHST15 antibody (upper panel) and H\&E (lower panel) are depicted. The negative control was stained using an identical procedure but with the primary antibody omitted. CHST15 staining status was assessed as (A) control, (B) negative, (C) weak, (D) moderate or (E) strong. H\&E staining of the same areas of (F) control, (G) negative, (H) weak, (I) moderate and (J) strong tissue. Scale bars, $200 \mu \mathrm{m}$; magnification, x100. CHST15, carbohydrate sulfotransferase 15; PDA, pancreatic ductal adenocarcinoma; H\&E, hematoxylin and eosin.

expression: Strong overexpression in PDA tissue, and negative-to-moderate expression in PDA tissue.

The results of the present study demonstrated that CHST15 overexpression was significantly associated with DFS and OS. Patients with PDA with overexpressed CHST15 had significantly worse DFS and OS rates than patients with negative-to-moderate CHST15 expression. In addition, there were no differences in sex, age at surgery, tumor location, tumor stage, tumor size, or tumor pathology between the strong and negative-to-moderate CHST15 expression groups. Therefore, CHST15 may be a recurrence and prognostic marker and a candidate therapeutic target in patients with PDA following surgical resection. In a previous study, CHST15 double stranded RNA that selectively inhibits CHST15 genes was injected in 4 patients with non-resectable PDA through endoscopic ultrasonography (29). In the clinical trial, reduction of tumor size was detected. Notably, CHST15 protein and partial necrosis were also detectable in biopsy specimens. Furthermore, the serum CD44 variant and CA19-9 levels decreased. These results suggested that CHST15 may be a novel target for inoperable patients and patients following surgical resection to prevent early recurrences.

CS-E is known to promote tumor invasion by cleaving CD44 in PDA tissue $(17,20)$. In the present study, serum samples were not available to assess soluble CD44. Therefore, the association of CD44 expression with DFS and OS was analyzed in PDA tissue obtained from surgical specimens. Unexpectedly, it was not possible to identify high expression of CD44 as a prognostic factor for patients with PDA following surgical resection. The association of CD44 expression levels with cancer prognosis and the utility of CD44 as a cancer stem cell marker have been debated. In the present study, a significant association between CD44 and CHST15 expression levels in PDA tissue was detected. However, certain PDA cells that expressed high CHST15 levels did not exhibit CD44 expression. This may be a reason, at least in part, why it was not possible to detect high CD44 expression as a prognostic marker for patients with PDA following surgical resection. As CD44 cleavage is enhanced by the CS-E fragment, which is synthesized by CHST15 (17), the levels of soluble CD44 in serum may be more closely associated with CHST15 expression intensity. The CS-E fragment
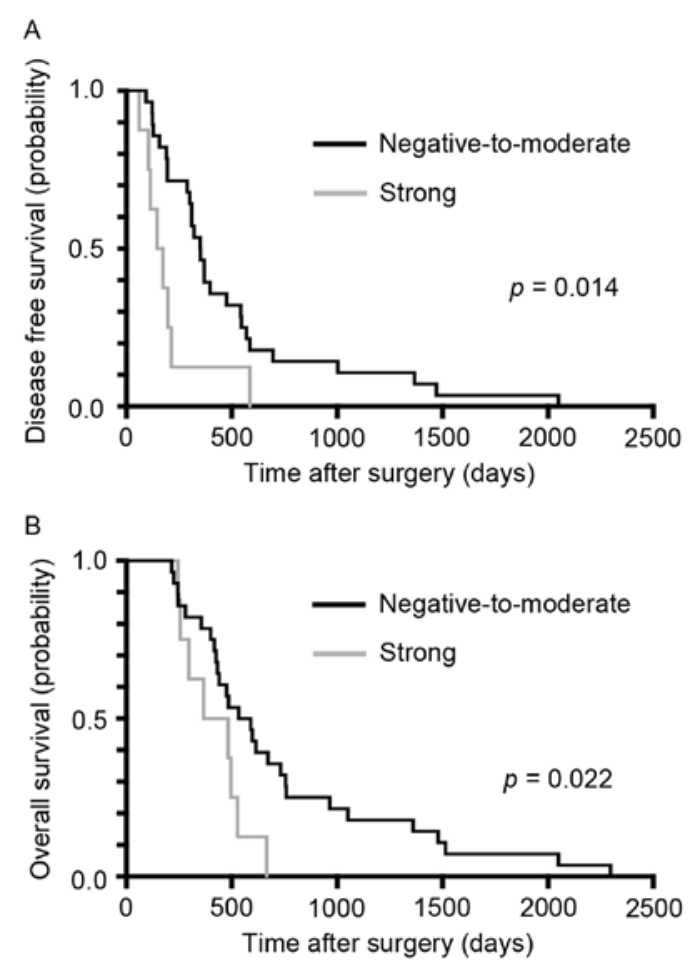

Figure 2. OS and DFS in patients with PDA. Kaplan-Meier curves stratified by carbohydrate sulfotransferase 15 expression and (A) DFS and (B) OS in patients with PDA following surgical resection. DFS, disease free survival; OS, overall survival; PDA, pancreatic ductal adenocarcinoma.

modulates tumor cell adhesion and migration by interacting with CD44 on tumor cells, leading to CD44-mediated tumor progression (17). Therefore, CD44 cleavage levels in patients with PDA may be a prognostic factor following surgical treatment. The exact mechanism of CS-E fragment production in vivo and the association with CD44 remain unclear. Further studies are required to evaluate the clinical significance of CHST15 and CD44 as prognostic factors in a large sample of patients with PDA.

Several previous reports have indicated that low NLRs are also associated with significantly improved prognoses in patients with PDA. Therefore, NLR may be a novel marker 
Table IV. Characteristics of patients with strong and negative-to-moderate CHST15 expression.

\begin{tabular}{|c|c|c|c|}
\hline CHST15 intensity & Strong & Negative-to-moderate & P-value \\
\hline Age at surgery (years) & & & N.S. \\
\hline$\geq 65$ & $7(87.5 \%)$ & $17(60.7 \%)$ & \\
\hline$<65$ & $1(12.5 \%)$ & $11(39.3 \%)$ & \\
\hline Sex & & & N.S. \\
\hline Male & $3(37.5 \%)$ & $17(60.7 \%)$ & \\
\hline Female & $5(62.5 \%)$ & $11(39.3 \%)$ & \\
\hline Tumor location & & & N.S. \\
\hline Head & $7(87.5 \%)$ & $23(82.1 \%)$ & \\
\hline Body-to-tail & $1(12.5 \%)$ & $5(17.9 \%)$ & \\
\hline Pathology & & & N.S. \\
\hline Well-to-moderate & $6(75 \%)$ & $22(78.6 \%)$ & \\
\hline Poor & $2(25 \%)$ & $6(21.4 \%)$ & \\
\hline Tumor stage & & & N.S. \\
\hline $\mathrm{I} / \mathrm{II}$ & $8(100 \%)$ & $25(89.3 \%)$ & \\
\hline III/IV & $0(0 \%)$ & $3(10.7 \%)$ & \\
\hline Tumor size & & & N.S. \\
\hline$<3 \mathrm{~cm}$ & $2(25 \%)$ & $8(28.6 \%)$ & \\
\hline$\geq 3 \mathrm{~cm}$ & $6(75 \%)$ & $20(71.4 \%)$ & \\
\hline Leukocytes (counts/ $\mu \mathrm{l}$ ) & $5,200(4375-7275)$ & $5,850(4725-8275)$ & N.S. \\
\hline Neutrophils (counts $/ \mu 1$ ) & $2,850(2525-5175)$ & $3,950(2950-6625)$ & N.S. \\
\hline Lymphocytes (counts/ $\mu \mathrm{l}$ ) & 1,400 (1300-1900) & $1,200(925-1400)$ & N.S. \\
\hline $\operatorname{NLR}(\%)$ & $1.75(1.25-3.94)$ & $4.21(2.23-5.28)$ & N.S. \\
\hline CRP (mg/dl) & $0.10(0.10-0.25)$ & $0.2(0.10-1.15)$ & N.S. \\
\hline Hemoglobin $(\mathrm{g} / \mathrm{dl})$ & $12.35(11.10-13.13)$ & $12.50(11.53-13.98)$ & N.S. \\
\hline Platelet (x10 counts $/ \mu 1)$ & $20.85(15.95-24.83)$ & $24.25(19.10-30.05)$ & N.S. \\
\hline Albumin (g/dl) & $3.75(3.63-3.80)$ & $3.95(3.60-4.30)$ & N.S. \\
\hline CA19-9 (U/ml) & $70.50(14.0-171.75)$ & $103.0(45.75-382.50)$ & N.S. \\
\hline
\end{tabular}

Variables are presented as N (\%) or the median (25-75th percentile). CHST15, carbohydrate sulfotransferase 15; NLR, neutrophil-to-lymphocyte ratio; CRP, C-reactive protein; CA19-9, carbohydrate antigen 19-9; N.S, not significant.

(A)

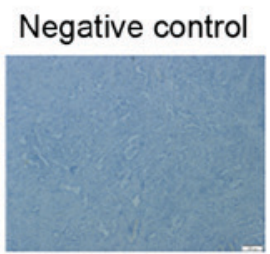

(F)

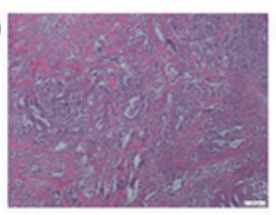

(B)
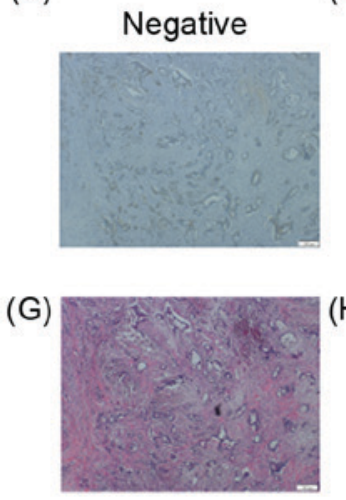

(C)
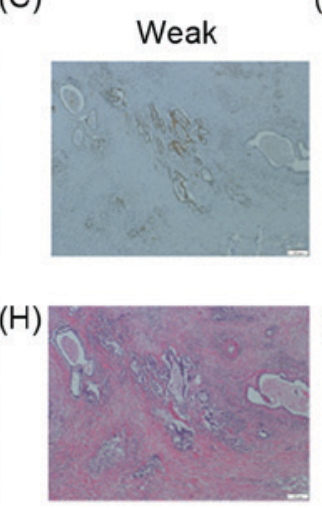

(D)
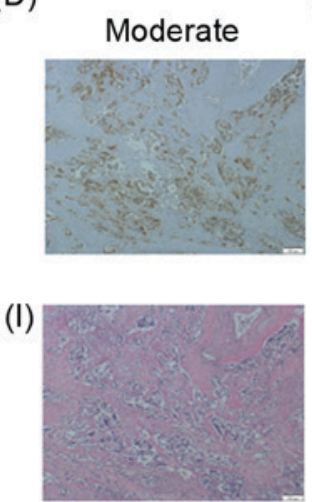

$(\mathrm{E})$
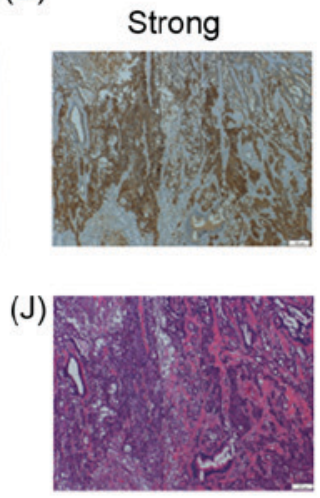

Figure 3. CD44 intensity in PDA tissue. Representative images of PDA tissue stained with CD44 (upper panel) and H\&E (lower panel) are depicted. The negative control was stained using an identical procedure but with the primary antibody omitted. CD44 staining status was assessed as (A) control, (B) negative, (C) weak, (D) moderate or (E) strong. H\&E staining of the same areas of (F) control, (G) negative, (H) weak, (I) moderate and (J) strong tissue. Scale bars, $200 \mu \mathrm{m}$; x100 magnification. PDA, pancreatic ductal adenocarcinoma; H\&E, hematoxylin and eosin.

for survival $(23,24)$. As expected, NLR was also identified as a prognostic factor in patients with PDA following surgical resection. In addition, a previous report indicated that a high NLR was significantly associated with tumor metastasis, poor 
tumor differentiation and high CA19-9, low Alb and high CRP levels (23). In the present study, low Alb levels and pathology were also associated with OS. However, neither CA19-9 nor CRP levels were associated with DFS or OS in the present study.

The results of the present study reinforced the importance of CHST15 as a prognostic factor in patients with PDA following surgical resection. Further investigation of CHST15 and its interaction with CD44 may provide novel insights into the manner in which tumor recurrence is regulated. PDA remains a lethal disease with a high early mortality rate following surgical therapy. To overcome early mortality following surgical resection, the analysis of mechanisms of CHST15 in tumor progression may lead to the development of alternative treatments that are able to control early recurrence.

\section{Acknowledgements}

The present study was supported, in part, by Grants-in-Aid for Scientific Research (C) from the Ministry of Education, Cultures, Sports, Science and Technology of Japan (grant no. $15 \mathrm{~K} 09050)$.

\section{References}

1. Jemal A, Siegel R, Xu J and Ward E: Cancer statistics, 2010. CA Cancer J Clin 60: 277-300, 2010.

2. Stathis A and Moore MJ: Advanced pancreatic carcinoma: Current treatment and future challenges. Nat Rev Clin Oncol 7: 163-172, 2010.

3. Chari ST, Kelly K, Hollingsworth MA, Thayer SP, Ahlquist DA, Andersen DK, Batra SK, Brentnall TA, Canto M, Cleeter DF, et al: Early detection of sporadic pancreatic cancer: Summative review. Pancreas 44: 693-712, 2015.

4. Arslan C and Yalcin S: Current and future systemic treatment options in metastatic pancreatic cancer. J Gastrointest Oncol 5: 280-295, 2014.

5. Fischer R, Breidert M, Keck T, Makowiec F, Lohrmann C and Harder J: Early recurrence of pancreatic cancer after resection and during adjuvant chemotherapy. Saudi J Gastroenterol 18: 118-121, 2012.

6. Pothacharoen P, Siriaunkgul S, Ong-Chai S, Supabandhu J, Kumja P, Wanaphirak C, Sugahara K, Hardingham T and Kongtawelert P: Raised serum chondroitin sulfate epitope level in ovarian epithelial cancer. J Biochem 140: 517-524, 2006.

7. Vallen MJ, Schmidt S, Oosterhof A, Bulten J, Massuger LF and van Kuppevelt TH: Primary ovarian carcinomas and abdominal metastasis contain 4,6-disulfated chondroitin sulfate rich regions, which provide adhesive properties to tumour cells. PLoS One 9: e111806, 2014

8. Ricciardelli C, Mayne K, Sykes PJ, Raymond WA, McCaul K, Marshall VR, Tilley WD, Skinner JM and Horsfall DJ: Elevated stromal chondroitin sulfate glycosaminoglycan predicts progression in early-stage prostate cancer. Clin Cancer Res 3: 983-992, 1997.

9. Ricciardelli C, Quinn DI, Raymond WA, McCaul K, Sutherland PD, Stricker PD, Grygiel JJ, Sutherland RL, Marshall VR, Tilley WD and Horsfall DJ: Elevated levels of peritumoral chondroitin sulfate are predictive of poor prognosis in patients treated by radical prostatectomy for early-stage prostate cancer. Cancer Res 59: 2324-2328, 1999.

10. Vallen MJ, van der Steen SC, van Tilborg AA, Massuger LF and van Kuppevelt TH: Sulfated sugars in the extracellular matrix orchestrate ovarian cancer development: 'when sweet turns sour'. Gynecol Oncol 135: 371-381, 2014.

11. van der Steen SC, van Tilborg AA, Vallen MJ, Bulten J, van Kuppevelt TH and Massuger LF: Prognostic significance of highly sulfated chondroitin sulfates in ovarian cancer defined by the single chain antibody GD3A11. Gynecol Oncol 140: 527-536, 2016.
12. ten Dam GB, van de Westerlo EM, Purushothaman A, Stan RV, Bulten J, Sweep FC, Massuger LF, Sugahara K and van Kuppevelt TH: Antibody GD3G7 selected against embryonic glycosaminoglycans defines chondroitin sulfate-E domains highly up-regulated in ovarian cancer and involved in vascular endothelial growth factor binding. Am J Pathol 171: 1324-1333, 2007.

13. Wegrowski Y and Maquart F: Chondroitin sulfate proteoglycans in tumor progression. Adv Pharmacol 53: 297-321, 2006.

14. Mizumoto S, Watanabe M, Yamada S and Sugahara K: Expression of $\mathrm{N}$-acetylgalactosamine 4-sulfate 6-O-sulfotransferase involved in chondroitin sulfate synthesis is responsible for pulmonary metastasis. Biomed Res Int 2013: 656319, 2013.

15. Ohtake $S$, Ito $Y$, Fukuta $M$ and Habuchi O: Human $\mathrm{N}$-acetylgalactosamine 4-sulfate 6-O-sulfotransferase cDNA is related to human $\mathrm{B}$ cell recombination activating gene-associated gene. J Biol Chem 276: 43894-43900, 2001.

16. Takakura K, Shibazaki Y, Yoneyama H, Fujii M, Hashiguchi T, Ito Z, Kajihara M, Misawa T, Homma S, Ohkusa T and Koido S: Inhibition of cell proliferation and growth of pancreatic cancer by silencing of carbohydrate sulfotransferase 15 in vitro and in a Xenograft model. PLoS One 10: e0142981, 2015.

17. Sugahara KN, Hirata T, Tanaka T, Ogino S, Takeda M, Terasawa H, Shimada I, Tamura J, ten Dam GB, van Kuppevelt TH and Miyasaka M: Chondroitin sulfate E fragments enhance CD44 cleavage and CD44-dependent motility in tumor cells. Cancer Res 68: 7191-7199, 2008.

18. Thomas L, Byers HR, Vink J and Stamenkovic I: CD44H regulates tumor cell migration on hyaluronate-coated substrate. J Cell Biol 118: 971-977, 1992.

19. Günthert AR, Sträter J, von Reyher U, Henne C, Joos S, Koretz K, Moldenhauer G, Krammer PH and Möller P: Early detachment of colon carcinoma cells during CD95 (APO-1/Fas)-mediated apoptosis. I. De-adhesion from hyaluronate by shedding of CD44. J Cell Biol 134: 1089-1096, 1996.

20. Goebeler M, Kaufmann D, Bröcker EB and Klein CE: Migration of highly aggressive melanoma cells on hyaluronic acid is associated with functional changes, increased turnover and shedding of CD44 receptors. J Cell Sci 109: 1957-1964, 1996.

21. Hirata K, Suzuki H, Imaeda H, Matsuzaki J, Tsugawa H, Nagano O, Asakura K, Saya H and Hibi T: CD44 variant 9 expression in primary early gastric cancer as a predictive marker for recurrence. Br J Cancer 109: 379-386, 2013.

22. Cho Y, Lee HW, Kang HG, Kim HY, Kim SJ and Chun KH: Cleaved CD44 intracellular domain supports activation of stemness factors and promotes tumorigenesis of breast cancer. Oncotarget 6: 8709-8721, 2015.

23. Yang JJ, Hu ZG, Shi WX, Deng T, He SQ and Yuan SG: Prognostic significance of neutrophil to lymphocyte ratio in pancreatic cancer: A meta-analysis. World J Gastroenterol 21: 2807-2815, 2015

24. Takakura K, Ito Z, Suka M, Kanai T, Matsumoto Y, Odahara S, Matsudaira H,Haruki K,Fujiwara Y,Saito R, et al: Comprehensive assessment of the prognosis of pancreatic cancer: Peripheral blood neutrophil-lymphocyte ratio and immunohistochemical analyses of the tumour site. Scand J Gastroenterol 51: 610-617, 2016.

25. Edge SB and Compton CC: The American Joint Committee on Cancer: The 7th edition of the AJCC cancer staging manual and the future of TNM. Ann Surgi Oncol 17: 1471-1474, 2010.

26. Metindir J, Dilek GB and Pak I: Staining characterization by immunohistochemistry of tumor cancer antigen in patients with endometrial cancer. Eur J Gynaecol Oncol 29: 489-492, 2008.

27. Lara MF, González-González E, Speaker TJ, Hickerson RP, Leake D, Milstone LM, Contag CH and Kaspar RL: Inhibition of CD44 gene expression in human skin models, using self-delivery short interfering RNA administered by dissolvable microneedle arrays. Hum Gene Ther 23: 816-823, 2012.

28. Kugelman LC, Ganguly S, Haggerty JG, Weissman SM and Milstone LM: The core protein of epican, a heparan sulfate proteoglycan on keratinocytes, is an alternative form of CD44. J Invest Dermatol 99: 886-891, 1992.

29. Nishimura M, Yahagi N, Itoi T, Ochiai Y and Matsuda Y: A translational study to investigate the role of carbohydrate sulfotransferase 15 for pancreatic cancer biology from in vitro to first-in-human clinical research. J Clin Oncol 33: e22201, 2015. 